\title{
Gobernanza ambiental en cuencas transfronterizas: la cuenca del río Sixaola (Costa Rica-Panamá)
}

\section{Environmental governance in transboundary basins: The Sixaola River Basin (Costa Rica-Panamá)}

\author{
Tania Rodríguez Echavarría \\ Escuelas de Ciencias Políticas y Geografía, Universidad de Costa Rica, San José, Costa Rica \\ tania.rodriguezechavarria@ucr.ac.cr \\ ORCID: https://orcid.org/o0oo-000I-5476-I575 \\ ISSN-OI85-4259; e- ISSN: 2007-9I76 \\ DoI: http://dx.doi.org/I0.28928/ri/872019/atc3/rodriguezechavarriat
}

\begin{abstract}
Resumen
La cuenca del río Sixaola es considerada por las organizaciones internacionales como una región fronteriza propensa para desarrollo de proyectos que promuevan la gobernanza ambiental. En este contexto, con el apoyo de la Unión Internacional para la Conservación de la Naturaleza (UICN) y el Banco Interamericano de Desarrollo (BID) se promovió la conformación de la Comisión Binacional de la Cuenca del Sixaola, una plataforma multi-actor (PMA) que buscaba promover la gestión integrada de la cuenca. Sin embargo, el presente artículo busca analizar su arquitectura, representatividad y legitimidad. Esto en razón de que esta comisión ha sido cuestionada, por un lado, porque en ella no participan la totalidad de los actores claves de la cuenca y, por otro lado, porque en su agenda política no se incorpora una de las principales problemáticas ambientales de esta frontera: la contaminación y la expansión de monocultivos de banano y plátano. ¿Es esta PMA una estructura que fomente la gobernanza de estos recursos compartidos? Palabras clave: frontera, plataformas multi-actores, ambiente, cuencas
\end{abstract}

\footnotetext{
Abstract

The Sixaola river basin is considered by international organizations as a border region prone to the development of projects that promote environmental governance. In this context, with the support of the International Union for Conservation of Nature (IUCN) and the Inter-American Development Bank (IDB), the creation of the Sixaola Basin Binational Commission was promoted, a multi-stakeholder platform (PMA) that sought to promote the integrated management of the basin. However, this article seeks to analyze its architecture, representativeness and legitimacy. This is due to the fact that this commission has been questioned, on the one hand, due to the fact that not all the key actors of the basin participate in it and, on the other hand, because its political agenda does not incorporate one of the main environmental problems of this border: the contamination and expansion of banana and plantain monocultures. Is this multi-actor platform a structure that fosters the governance of these shared resources?

Key words: Border, Multi-stakeholder platforms, environment, basins,
}

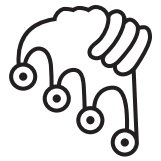

IZTAPALAPA

Agua sobre lajas 


\section{Introducción}

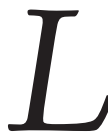

as fronteras centroamericanas, siguiendo la tradición de la corona española, se caracterizan por haber sido trazadas usando como referencia cursos de agua, los cuales fueron usados durante la colonia para dividir las provincias y después, con la independencia, las fronteras de los nacientes Estados. En esta región existen 24 cuencas hidrográficas compartidas entre dos o más Estados. Las cuencas transfronterizas representan $36 \%$ del territorio centroamericano y concentran las principales reservas de agua dulce del istmo (Kauffer, 2006: 10).

Las regiones fronterizas en Centroamérica se caracterizan por ser rurales, poco pobladas, de difícil acceso y han estado marcadas por la débil presencia e inversión estatal (Rodríguez, 20I4). La falta de vigilancia por parte de instituciones de gobierno ha facilitado que en ellas prevalezcan condiciones sin regulación ni control, y eso ha permitido la expansión tanto de prácticas extractivas como de actividades ilícitas, entre ellas el crimen organizado y el narcotráfico, como ha sido el caso de la Reserva de la Biosfera Maya y de otros territorios rurales de Centroamérica (McSweeney, Richani, Pearson, Devine y Wrathall, 2017).

La importancia ambiental y geopolítica de estas regiones transfronterizas motivó, a partir de la década de 1990 y después de los procesos de paz en Centroamérica, que una gran diversidad de actores endógenos (nacionales y locales) y exógenos (internacionales y trasnacionales) promovieran paulatinamente la firma de acuerdos, proyectos y programas que buscaban principalmente el desarrollo y la conservación de estas regiones. ${ }^{1}$ Es así como se han puesto en marcha importantes iniciativas

1 Un importante ejemplo es el acuerdo firmado en 1995 por los vicepresidentes centroamericanos para el "Desarrollo sostenible fronterizo", elaborado gracias al apoyo brindado por la Organización de Estados Americanos (oEA), la Secretaría de Integración Económica Centroamericana (SIECA) y la Fundación para la Paz y a Democracia (FunDPADEM), el cual ve el desarrollo de las zonas fronterizas como un instrumento para reforzar los lazos entre los países y por consiguiente su integración (Medina, 2009:42). 
gubernamentales, desarrolladas por la cooperación internacional, que buscan promover la cooperación transfronteriza con el fin de conservar estos recursos compartidos.

En la cuenca del río Sixaola, región fronteriza dinámica entre Costa Rica y Panamá, se concentra una biodiversidad importante, con riqueza de recursos naturales y un curso de agua utilizado para riego, transporte y para marcar el segmento fronterizo localizado en la vertiente Caribe. Junto a la riqueza natural y cultural (presencia de pueblos indígenas y de poblaciones afrocaribeñas) de esta cuenca se localiza el cantón de Talamanca, uno de los más pobres de Costa Rica, con uno de los índices de desarrollo más bajos del país. En 2014 estaba en la posición 80 de los 8 I cantones existentes en el país con un índice de desarrollo humano (IDH) de 0.634 (PNUD Y UCR, 20I6). ${ }^{2}$

Es importante aclarar aquí que esta investigación se inscribe en la línea de estudios que analizan las fronteras como un objeto geográfico y como una expresión de lo político (Anne Laure Amilhat-Szary y Fourny, 2006; Fourny, 2005; Medina, 2009). Las fronteras son concebidas no como líneas sino como regiones dinámicas susceptibles de integrar territorios a pesar de la discontinuidad política que impone el trazado fronterizo. Alrededor de las fronteras se articulan una gran diversidad de actores, de ecosistemas y de actividades productivas y se ponen en práctica relaciones de fuerza como en todo territorio, pero complejizadas por la cercanía de dos sistemas políticos diferentes (Rodríguez, 20I4).

Entiendo por cuenca hidrográfica, según la definición de Aguilar et Iza (2009), como el perímetro delimitado por el escurrimiento de las aguas superficiales y subterráneas constituidos por ríos, acuíferos y tierras, humedales cuya su delimitación geográfica se hace en función de las zonas de influencia hidrográfica (Aguilar \& Iza, 2009).

El presente artículo tiene por objetivo en primer lugar dilucidar cómo se ha tratado de promover la gobernanza de estas cuencas a través de iniciativas de cooperación para la conservación del ambiente, pensadas e implementadas por organizaciones internacionales como el Banco Interamericano de Desarrollo y la Unión Internacional para la Conservación de la Naturaleza (UICN), entre otros. Iniciativas que tienen en común el interés por promover la gestión integrada de la cuenca de río Sixaola a través de la gobernanza ambiental y la creación de comisiones binacionales.

2 Atlas del desarrollo bumano cantonal de Costa Rica 2016, realizado por el Programa de las Naciones Unidas para el Desarrollo y la Universidad de Costa Rica http://desarrollohumano.or.cr/mapa-cantonal/index.php 
Entenderemos por gobernanza ambiental una forma de ejercicio del poder que promueve la participación horizontal de actores privados y públicos con el fin de gestionar deliberativamente la naturaleza. Esta gobernanza se ha venido poniendo en práctica por medio de diversos dispositivos tanto normativos como deliberativos que estudiaremos en este artículo.

De esta forma se tratará de clarificar cómo se ha venido adoptando esta gobernanza ambiental y el rol que ha tenido la Comisión Binacional de la Cuenca del Sixaola como una plataforma multiactor que articula agentes tanto públicos como privados con el fin de gestionar de forma "participativa" esta cuenca. Nos interesaremos en el accionar de esta comisión, en particular en cómo se fija la agenda política, quiénes participan y por qué. Además, nos cuestionaremos sobre la percepción que tienen de esta Comisión los demás actores que coexisten en esta cuenca. Analizaremos particularmente sus logros, su representatividad, legitimidad y la efectividad de sus decisiones.

\section{Metodología}

Recuperamos resultados de un largo proceso de investigación que inició en el 2010 en el marco de una tesis doctoral y que se ha convertido en dos proyectos. El primero ha venido estudiando los procesos de cooperación y de conflicto que se dan alrededor de la gestión ambiental de la cuenca del Sixaola y el segundo analiza la pertinencia de la gestión por cuenca y de la gobernanza del agua como estrategias de intervención en los territorios. Estos dos proyectos desarrollados desde la Universidad de Costa Rica, a partir de 20I5, han permitido la realización de alrededor 40 entrevistas a profundidad a actores locales, a gestores de proyectos de cooperación y a autoridades locales y nacionales.

Paralelamente a las entrevistas se ha generado un proceso de observación participante en las reuniones y actividades de la Comisión Binacional Costa Rica-Panamá con el fin de comprender su funcionamiento y su agenda política.

\section{La cuenca del río Sixaola, de enclave bananero a un laboratorio de la gobernanza ambiental}

La cuenca del río Sixaola compartida entre Costa Rica y Panamá es una región fronteriza dinámica que cubre alrededor de $2839.6 \mathrm{~km}^{2}$ (Franklin, 2007). La parte 
alta está poblada en $95 \%$ por grupos indígenas bribri, cabécar y ngóbe buglé y la parte media y baja por mestizos y afrodescendientes.

Cabe destacar que esta región ha estado marcada por el abandono por parte de los gobiernos centrales, lo que facilitó la instalación, desde el siglo xIx, de la empresa trasnacional estadounidense United Fruit Company, la cual se benefició de las debilidades estructurales de este territorio para desarrollar sistemas de plantación basados en la lógica del enclave (Girot y Granados, 1997).

Es importante precisar que la lógica de enclave impuesta por la empresa bananera se puede asimilar al régimen de apartheid que consistía en una segregación en dos niveles, uno interno y otro entre el enclave como estructura de producción y el resto del país. De esta forma, los enclaves se caracterizaban por estar aislados y tener sus propias autoridades (Duncan y Melendez, 1972). Si bien estas comunidades fueron progresivamente incorporadas al sistema político costarricense a partir de la década de los sesenta, todavía siguen estando mal integradas y en ellas se puede observar los resabios de la lógica de enclave. Hoy en día, por ejemplo, muchos de los poblados fronterizos mantienen la organización de los antiguos cuadrantes bananeros y los nombres de las fincas como "Finca 6o" en Changuinola (Panamá) o "Finca 98" en Sixaola (Costa Rica) (Rodríguez, 2014: 169). Además, el cultivo de banano y plátano sigue siendo la actividad productiva más importante en estos territorios y es realizada por empresas también trasnacionales herederas de la United Fruit Company (ufCo), como Dole, Del Monte y Chiquita.

Es así como la UfCo tuvo un papel central en la precisión del trazado de la frontera y en su configuración territorial. Se pudo constatar durante las entrevistas que la producción de monocultivos, en especial de banano, está naturalizada en el imaginario de las personas residentes, las cuales manifestaron que: "[el banano] siempre ha estado ahí".

Paralelamente a estas actividades extractivas, la riqueza cultural y ambiental de esta cuenca ha generado que en ella coexistan también diferentes tipos de categorías de manejo del territorio como lo son la zona marítimo-terrestre, los territorios indígenas, las áreas protegidas (Parque Internacional La Amistad, refugios de vida silvestre y parques nacionales) y la zona fronteriza. Aunado a esto, en este territorio conviven asentamientos campesinos, grandes propietarios de tierra, mayoritariamente extranjeros, que son además empresarios turísticos (Llaguno, Solano, Espeleta, Alfaro y Moraga, 2014).

Es importante destacar que más de la mitad del territorio (55\%) corresponde a zonas de protección ambiental y $30 \%$ a territorios indígenas. Es así como alrededor de $85 \%$ del territorio de Talamanca está bajo alguna categoría de manejo, lo que ha 
generado importantes tensiones en los límites de estos territorios, ya que muchas veces se presentan conflictos con proyectos turísticos y extractivos que buscan expandirse (Llaguno et al., 2014).

A diferencia de otras regiones fronterizas centroamericanas, esta cuenca es una región pacífica y propicia para la cooperación transfronteriza, lo que ha facilitado el desarrollo de todo un marco legal de cooperación que ha sido la base de numerosos proyectos y programas de cooperación que buscaban principalmente el desarrollo fronterizo y la conservación del ambiente.

MAPA I.

Localización de la cuenca del río Sixaola

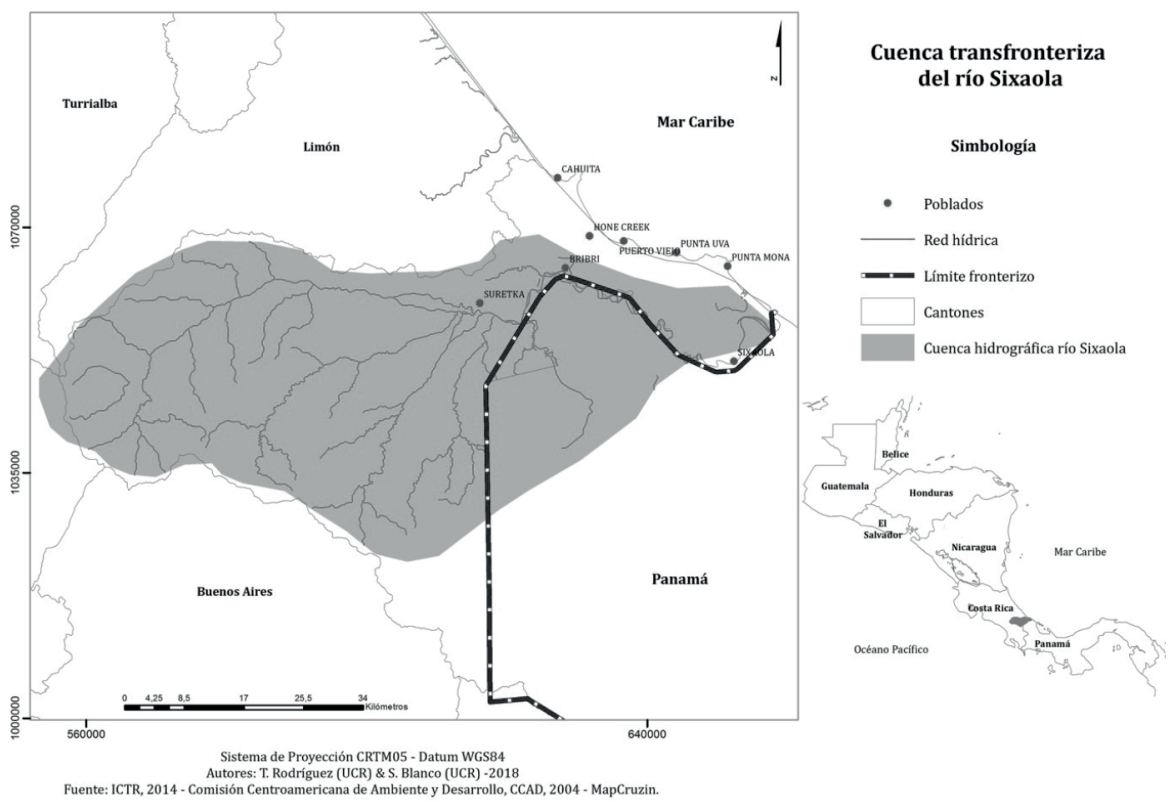

Desde la década de los noventa, organizaciones como la Unión Internacional para la Conservación de la Naturaleza (UICN), The Nature Conservancy (TNC), el World Wildlife Fund (WWF), así como organismos intergubernamentales (oIG) como el Banco Interamericano de Desarrollo (BID) ${ }^{3}$ han identificado la cuenca del río

3 El BID como el Banco Mundial, a partir de la década de los ochenta, vivió un "enverde- 
Sixaola como una región transfronteriza estratégica, en razón de su importante biodiversidad (Franklin, 2007).

Esta frontera es percibida ${ }^{4}$ especialmente por los representantes de ONG internacionales como una zona de "oportunidad" que aporta significativas ventajas al momento de ejecutar un proyecto de cooperación. Ante todo, han manifestado que trabajar en zonas fronterizas permite a un solo proyecto tener impactos en dos países a la vez. Condición que es vista como positiva por los principales donantes que evalúan los proyectos (Cooperación Noruega, Dinamarca, Ministerio de Ambiente Alemán, usAID). Además, Costa Rica y Panamá cuentan con el marco de "cooperación transfronteriza más desarrollado de la región centroamericana" (Girot y Granados, 1997). ${ }^{5}$ Representantes de organizaciones como la UICN, TNC y RUTA ${ }^{6}$ afirmaron durante las entrevistas que, junto con su importancia ambiental, el hecho de que esta frontera fuera considerada como una de las más pacíficas del istmo y anuente a la cooperación fue un factor determinante para escogerla como sitio para

cimiento" que implicó su reorientación y la creación del Fondo para el Medio Ambiente Mundial (FMAM), de donde provienen los recursos que financian el Proyecto para la gestión integrada de ecosistemas de la cuenca binacional del río Sixaola (BID GEF).

Los proyectos financiados por el BID son sumamente complejos desde su concepción hasta su ejecución, ya que son préstamos o donaciones que provienen de los Estados. Para el caso de la cuenca del río Sixaola hay dos proyectos de este tipo: I. Proyecto para la gestión integrada de ecosistemas de la cuenca binacional del río Sixaola. Este proyecto es una donación, es binacional y es conocido por los actores locales como el bid Gef (siglas en el inglés del Fondo para el Medio Ambiente Mundial [fmam]). 2. Programa para el Desarrollo Sostenible de la cuenca del Sixaola, el cual es un préstamo que solo se aplica en Costa Rica y es conocido por los actores locales como el BID MAG.

4 Forma parte de los resultados generados por el proceso de investigación (entrevistas y análisis de contenido de los documentos de proyecto).

5 Esto es el caso del "Convenio para el Desarrollo Fronterizo Costa Rica-Panamá", firmado en 1992 pero que no entra en vigencia hasta 1995. Este convenio establece una Comisión Binacional Permanente como órgano de decisión y una Secretaría Ejecutiva con representantes en cada uno de los países. La Comisión es la responsable de darle seguimiento a las actividades y a los acuerdos generados.

6 La Unidad Regional de Asistencia Técnica (RUTA) es una iniciativa conjunta intergubernamental de los siete países centroamericanos (Belice, Costa Rica, El Salvador, Guatemala, Honduras, Nicaragua y Panamá) y de siete agencias internacionales de desarrollo (MAEC, FIDA, ADA, BID, FAO, IFPRI, IICA ) para fomentar el desarrollo sostenible y la reducción de la pobreza en las áreas rurales de Centroamérica http://www.ruta.org/ historia_de_ruta.php 
implementar sus proyectos. Muchos de estos actores estuvieron de acuerdo en que esta característica hace aún más viable la consecución de los objetivos planteados en estos proyectos.

\section{Gobernar el ambiente desde la Gestión Integrada del Recurso Hídrico (GIRH) y la cuenca}

A lo largo de la investigación se pudieron identificar proyectos que buscaban poner en práctica acciones de conservación alrededor de esta cuenca. El Proyecto para la gestión integrada de ecosistemas de la cuenca binacional del río Sixaola del BID GEF ${ }^{7}$ (200920I2), el Proyecto de Gobernanza en Cuencas Transfronterizas conocido como BRIDGE ${ }^{8}$ (Building River Dialogue and Governance-Construyendo Diálogo y Gobernanza en los Ríos) ${ }^{9}$ (20II-20I8) que quiere decir "puente" en español o el Proyecto Gobernanza y cambio climático en las cuencas transfronterizas (2010-2014), los dos de la Unión Internacional para la Conservación de la Naturaleza (UICN) o la Alianza Público Privada (2009-2013), conformada por el supermercado alemán REWE, las empresas Chiquita Land Company, $\operatorname{CorbanA}^{\mathbf{1 0}}$ y la agencia de Cooperación Alemanda (GIz), son solo algunos ejemplos de las iniciativas implementadas en esta cuenca.

Estas incitativas eligieron utilizar como unidad de gestión las cuencas transfronterizas. Además, todos estos proyectos recuperan los principios de la Gestión Integrada del Recurso Hídrico (GIRH), que también establecen las cuencas hidrográficas como unidad ideal para la gestión del agua. Además, incluyen elementos ligados a estrategias para promover la adaptación al cambio climático y promover mercados y actividades productivas verdes. Es interesante destacar que a pesar de que para los actores locales la principal problemática ambiental es la contaminación

7 Proyecto Rs-XIOI7 de la BID, del I2 de setiembre 2007 (documento disponible en el sitio del віD D). El Global Environmental Facility (GEF) es un fondo para la gestión del ambiente creado en 1990 para canalizar los financiamientos destinados a tratar los problemas ambientales globales. Participan ahí el Programa de las Naciones Unidas para el Desarrollo (PNUD), El Programa de las Naciones Unidas para el Medio Ambiente (pNuma) y en Banco Mundial.

8 Busca generar capacidades para la gobernanza en cuencas transfronterizas

9 IUCN, BRIDGE : Building River Dialogue and Governance, Proyecto, https://cmsdata. iucn.org/downloads/project_brief_bridge.pdf

${ }^{10}$ Empresa costarricense para la comercialización de banano. 
generada por los monocultivos de banano y plátano, este tema no es tratado en los proyectos estudiados.

Desde la década de los ochenta, redes internacionales y comunidades epistémicas del agua han estado promoviendo la GIRH, y con ella la gestión por cuenca. Una comunidad epistémica es una"red de profesionales que cuentan con expertise y competencias reconocidas en un área en particular que además pueden hacer valer un conocimiento considerado como pertinente en las políticas que tengan que ver con el área en cuestión" ${ }^{\prime 11}$ (Haas, 2013: 35I).

Estas comunidades de expertos tienen una creciente influencia ya sea en la creación de nuevas políticas o regulaciones, o en la difusión de ciertas ideas en la agenda política global ${ }^{12}$ (Meyer y Molyneux-Hodgson, 20IIः I4I). Es así como las comunidades epistémicas comparten competencias, métodos científicos y creencias normativas y tienen un papel central en los procesos a través de los cuales las nuevas ideas circulan de la sociedad hacia los gobiernos y de un país a otro (Bossy y Evard, 20Iо).

La GIRH aparece como concepto después de la Conferencia Internacional sobre el Agua y el Ambiente en Dublín en enero 1992, donde es promovida como la principal respuesta frente a los desafíos que enfrentaba la gestión del agua, debido a que esta se realizaba de forma fragmentada y poco coordinada entre sectores e instituciones (Julien, 2012: 5; Molle, 2012: 21). La declaración de Dublín establece cuatro principios que van a guiar "la gobernanza del agua y la implementación de la GIRH" (Julien, 20I2: 5) y declaran que el agua era un recurso frágil e indispensable para la vida y el desarrollo. Además, sostienen que es necesario promover la participación de usuarios, tomadores de decisiones y planificadores, así como reconocer el rol central de las mujeres en el abasto, la gestión y la preservación del agua.

También la GIRH admite el valor económico del agua. Este principio ha sido particularmente cuestionado ya que para muchos autores el agua se presenta como un "producto de mercado" cuyo costo debe tener un precio (Bouquet, 2012: 63). La suscripción a este principio permite evidenciar una toma de posición ideológica a escala internacional, en que se renuncia a declarar el agua como un bien público y universal.

11 "réseaux de professionnels ayant une expertise et une compétence reconnues dans un domaine particulier qui peuvent faire valoir un savoir pertinent sur les politiques du domaine en question»

12 "que se soit la création de nouvelles politiques ou de régulations, leur diffusion, ou le maintien de certaines idées sur l'agenda politique» 
Es interesante recalcar que la GIRH introduce la noción de cuenca hidrográfica como unidad ideal para la gestión del agua e insiste sobre la participación de los actores en los procesos deliberativos (Rodríguez, 2014:396). En la Cumbre de la Tierra, en Río, en junio de I992, la GIRH es definida como "un proceso que promueve la gestión y el desarrollo coordinado del agua, la tierra y los recursos relacionados, con el fin de maximizar el bienestar social y económico resultante de manera equitativa, sin comprometer la sostenibilidad de los ecosistemas" (GWP, 2000ः 24). Para F. Molle la GIRH engloba tres pilares claves: la eficiencia (racionalidad económica), la equidad (participación y gobernanza) y el ambiente (enfoque ecosistémico) (Molle, 20I2: 25).

Los proyectos de la UICN y el BID se basan en la GIRH y dentro de sus objetivos promueven la gestión integrada de la cuenca del Sixaola, por medio del fortalecimiento de la coordinación y cooperación institucional y de las capacidades de incidencia de la sociedad civil (autoridades indígenas, asociaciones de desarrollo y ONG locales) (Rodríguez, 20I4: 235). De esta forma la participación y la gobernanza en esta cuenca fueron dos preocupaciones centrales para estas organizaciones, por lo que dentro de sus proyectos proponen el desarrollo de una Comisión Binacional que gestione la cuenca del río Sixaola.

Es importante insistir sobre el hecho de que la GIRH es un proceso profundamente político, pensado en un contexto neoliberal que pone en duda el rol del Estado de bienestar, al cual juzga ineficaz, corrupto y un obstáculo para el desarrollo (Molle, 20I2: 25). En el sector del agua se adoptaron importantes reformas que implicaron el aumento de la participación del sector privado (especialmente los actores encargados de los servicios de agua como abastecimiento y saneamiento) con el fin de transferirles responsabilidades. Es así como desde la GIRH se promueve un sistema de gobernanza "efectiva y reactiva" (Molle, 20I2: 37) que releve al Estado.

En este contexto, la gobernanza se torna un concepto central que promueve el aumento de la participación de actores no estatales (empresas trasnacionales, ONG) y que valora mecanismos de toma de decisión "participativos" que buscan promover una democracia deliberativa más que una democracia representativa (Alphandery, Djama, Fortier y Fouilleux, 20I2: II).

La participación se vuelve una respuesta para lidiar con sistemas tan complejos y conflictivos como lo son las cuencas transfronterizas alrededor de las cuales se articulan una gran diversidad de actores con intereses muy diversos y que actúan a diferentes escalas (local, nacional, global). Es así que a través de estos proyectos y programas, la cuenca del Sixaola se transformó en un laboratorio de la cooperación (Rodríguez, 20I4:425) en donde se trató de poner en práctica procesos participativos 
con el fin de promover la buena gobernanza del agua y del ambiente para después ser replicados en otras cuencas a través del proyecto Bridge de la UiCN.

\section{¿Qué es la gobernanza ambiental?}

El estudio de estos proyectos de cooperación, permitió constatar rápidamente que la gobernanza es un concepto vago e impreciso, alrededor del cual se han desarrollado múltiples definiciones. La noción de gobernanza ha cambiado a lo largo del tiempo y ha suscitado múltiples debates. Para Guy Hermet, la palabra gobernanza es una especie de palabra "valija" que es utilizada para describir fenómenos muy diversos.

Inicialmente fue un concepto internacionalizado por el Banco Mundial desde la década de los años noventa como un mecanismo de gestión más eficaz y una nueva forma de ejercicio del poder que se sustentaba en la participación horizontal o no jerárquica de una gran diversidad de actores privados y públicos (Hermet, 2005). Pone en duda la eficiencia del Estado e introduce al actor privado en espacios de decisión sobre temas y bienes públicos. Cabe destacar que el actor privado es un actor colectivo cuya racionalidad está determinada por intereses privados, como es el caso de las empresas (Amilhat-Szary, 2009: 8).

La gobernanza se popularizó como un proceso que prometía "aumentar la democracia" (Papadopoulos, 20I2:3). Una especie de gobierno que iba más allá del Estado y que implicaba una recomposición de las formas tradicionales de autoridad política (Alphandery et al., 2012: 12), puesto que no considera al Estado y su organización territorial (gobiernos locales, entidades descentralizadas) como los únicos agentes de poder en el espacio (Amilhat-Szary, 2009: 7).

Se puede por lo tanto concluir que la gobernanza propone el abandono de la jerarquía público-privado, "un gobierno de las organizaciones y para las organizaciones”, sean estas públicas o privadas, cambiando así las relaciones de autoridad (Hermet, 2005: 35). En palabras de Amilhat-Szary la gobernanza desideologiza la separación tradicional entre lo público y lo privado y desacraliza el poder de lo público (Amilhat-Szary, 2009; Hermet, 2005) en función de principios de eficiencia y eficacia. Boaventura de Sousa (Santos, 2007) la llama "gobernanza neoliberal", la cual no debe confundirse con "la gobernanza contrahegemónica", que tiene más que ver con movimientos emancipatorios y formas de organización local de cogestión o autogestión, como lo son los Caracoles zapatistas. 


\section{Los dispositivos de gobernanza ambiental}

Los proyectos estudiados promueven a través de GIRH la gobernanza ambiental y para esto han desarrollado metodologías y estrategias de intervención en los territorios mediante dispositivos operativos que buscan manejar los recursos naturales.

Es así como estas instituciones y ONG internacionales ponen en práctica formas de participación horizontales entre actores con el fin de promover, al menos en el discurso, la negociación y la cooperación, términos muy utilizados cuando se habla de gobernanza (Chevallier, 2003: 109).

En el marco de la GIRH se concibieron múltiples instrumentos para materializar en los territorios los procesos de gobernanza. Estos instrumentos o estrategias, que denominaremos dispositivos de gobernanza, fueron difundidos por organizaciones intergubernamentales (OIG) y ONG internacionales a través de numerosos proyectos de cooperación (Rodríguez, 2014: 430).

Dore, Rebel y Molle analizan los instrumentos o dispositivos de gobernanza que se han venido desarrollando alrededor de la cuenca del río Mekong e identifican tres tipos:

I. Los instrumentos deliberativos como las plataformas multiactores

2. Los instrumentos técnicos (transmisión de conocimientos científicos en los procesos de toma de decisión)

3. Los instrumentos de incidencia política que buscan influenciar la opinión pública y a los tomadores de decisión, como los son las campañas de sensibilización que pueden apoyar o oponerse a una acción (Dore, Lebel y Molle, 20I2: 29)

Estos instrumentos en la realidad no se aplican de forma aislada, sino que muchas veces se utilizan de forma simultánea y son generalmente complementarios.

En el marco de esta investigación se pudo identificar específicamente el uso de dispositivos normativos (códigos o acuerdos) y deliberativos (como comités, plataformas multiactores o autoridades de cuenca) de gobernanza. Para efectos de este artículo nos centraremos en los dispositivos deliberativos, en especial en la Comisión Binacional para la Gestión de la Cuenca del río Sixaola. 


\section{Dispositivos deliberativos: las plataformas multiactores}

Las plataformas multiactores (PMA) son espacios de negociación, de participación voluntaria o formalizada que articulan a diferentes partes interesadas o stakeholders que debaten alrededor de un mismo problema o situación. Estas partes interesadas aceptan que son interdependientes, por lo que buscan una solución colectiva para solventar el problema común (Steins y Edwards, 1999: 244). Las PMA tienen una presencia habitual en los proyectos de cooperación ya que su creación es muchas veces solicitada por los organismos y agencias de cooperación que financian estos proyectos (Warner y Simpungwe, 2012: 108). En realidad constituyen un dispositivo deliberativo que requiere para su buen funcionamiento la participación de una pluralidad de actores públicos, privados y de la sociedad civil.

De esta forma son instituciones de negociación y/o de toma de decisiones, pueden ser foros, comités o comisiones formales o informales. Para muchos autores las PMA son, más que espacios de negociación, espacios de construcción colectiva de conocimientos que requieren para su buen funcionamiento contar con la participación de líderes locales reconocidos, ya que están siempre en busca de legitimidad (Dore et al., 2012: 7).

Muchas veces las PMA son vistas como herramientas potenciales para la resolución de conflictos en la gestión de recursos comunes (Warner y Simpungwe, 2012: I09). Idealmente pueden permitir que actores con intereses divergentes participen en un espacio en donde la mayoría sea la que determine la decisión, para así llegar a resultados representativos y justos (Warner y Simpungwe, 2012: 109). En teoría, estas PMA facilitarían la participación de poblaciones locales para que puedan presentar sus reivindicaciones. También muchas de estas pMA pueden ser espacios de oportunidad, ya que permiten que los actores locales interactúen con actores globales (oNG, oIG, empresas). Este es el caso de actores fronterizos que han tenido acceso a ayudas y a proyectos sin tener que pasar por las autoridades del gobierno central, que "históricamente los han ignorado" (Ghiotti, 2014: 17).

Para entender como funcionan las PMA se torna necesario analizar su arquitectura, es decir, identificar quién las crea, las convoca y las financia, así como su representatividad y legitimidad. 


\section{Arquitectura de la Comisión Binacional para la Gestión de la Cuenca del Sixaola}

Con el fin de entender cómo funcionan estas PMA, nos interrogamos sobre su arquitectura, es decir, su estructura, cómo y para qué fueron concebidas, así como sobre su funcionamiento.

Un primer resultado fue que las PMA estudiadas habían sido concebidas gracias a la intervención de actores exógenos con poca o nula participación de actores locales (Rodríguez, 20I4: 434). Tanto la UICN como por el BID y las alianzas público-privadas hacen uso de dispositivos deliberativos (comisiones, comités) para promover espacios "participativos" alrededor de la gestión del ambiente. Este es el caso de la Comisión Binacional para la Gestión de la Cuenca del Sixaola (CBGCs), creada en el 2009 en el marco del proyecto BID-GEF con el apoyo técnico de la UICN. Esta Comisión es una PMa top-dowm, ya que fue concebida por los secretarios del Convenio Binacional Costa Rica-Panamá, con la asistencia técnica de expertos provenientes del BID y del GEF. La Comisión articula actores gubernamentales como los representantes de los ministerios de ambiente, salud y agricultura de los dos países, instituciones como las Comisiones de Emergencia, así como otros sectores (cooperativas, sindicatos) y representantes de la sociedad civil, principalmente las autoridades indígenas.

Esta Comisión tiene por objetivo promover la gestión de la cuenca del Sixaola a través del enfoque propuesto por la GIRH y le da un importante espacio a la gestión del riesgo como un tema articulador. Desde su creación tenía por objetivo convertirse en el "órgano soberano" del proyecto BID-GEF y posteriormente de la cuenca, ya que fue concebida para que progresivamente se constituyera en una instancia de "gestión territorial” (Wong y Porras, 2013: 2). En este contexto la UICN, en el marco del proyecto BRIDGE, decide fortalecer esta PMA para que se convierta en "una entidad independiente del proyecto y potencialmente una unidad de planificación territorial y de gestión del desarrollo de la cuenca", es decir, una autoridad de cuenca (Wong y Porras, 20I3: 3). Para esto la UICN suscribe una alianza estratégica con el BID y los gobiernos de Costa Rica y de Panamá para así contribuir a la consolidación de esta Comisión, específicamente a través de la redacción de su reglamento interno. La redacción de este reglamento fue liderada por los expertos de la UICN, que realizaron talleres de consulta con actores clave. Es interesante constatar que este reglamento retoma principios e ideas que la UICN suscribe, como algunos principios del derecho internacional, especialmente los expuestos en la Convención de la oNU de 1997 sobre 
el derecho de los usos de los cursos de agua internacionales para fines distintos de la navegación.

El reglamento define a la Comisión como " una instancia binacional y transfronteriza de gobernanza para la gestión integrada de la cuenca binacional del río Sixaola" (Wong y Porras, 2013: 9). Ante esto, conviene rescatar que este reglamento no fue ni pensado ni reconocido por los gobiernos de Costa Rica y Panamá, por lo que es un acuerdo extraoficial suscrito solo por los miembros de la Comisión.

También es importante destacar la idea de que esta PMA ejecute acciones de cooperación que trasciendan los límites de los Estados, ya que se promueven acciones "transfronterizas"13 para conservar y gestionar ecosistemas compartidos, divididos por fronteras.

Al estudiar el funcionamiento de esta Comisión resulta evidente su importante dependencia tanto con respecto al apoyo técnico como al financiero de la UICN, ya que esta Comisión no posee fondos propios. La particularidad de estas regiones fronterizas, especialmente la falta de infraestructura y de transporte público, hace que los desplazamientos de los y las participantes sean muy caros y difíciles. Además, esta PMA se presenta como una instancia transfronteriza, lo que implica que las reuniones deben alternarse entre los dos países y esto a su vez supone tomar en cuenta los gastos que genera el pasar una frontera (transporte e impuestos). La UICN no solo paga el transporte y los viáticos de los y las participantes sino también la logística de la actividad (convocatoria, agenda, facilitación). Es así como la Comisión depende de los recursos y de la asistencia técnica de la UICN y del BID, lo cual pone en duda la sostenibilidad de esta instancia, ya que una vez que estos proyectos se retiren de los territorios estará en riesgo de desaparecer. Adicionalmente, diferentes actores locales entrevistados hicieron referencia a estos comités como "El comité de la UICN" о del "віD", lo que muestra una falta de apropiación por parte de los mismos miembros, que ven en esta estructura un espacio de participación artificial que fue "importada”. La falta de apropiación vulnerabiliza aún más la sostenibilidad en el tiempo de la Comisión.

13 Entenderemos por acciones transfronterizas aquellas que traspasan la frontera y generan impactos y dinámicas de cooperación o de conflicto independientemente de la línea divisoria. 


\section{Logros y retos de la Comisión Binacional para la Gestión de la Cuenca del Sixaola}

A pesar de la falta de recursos se ha logrado que los comités de cada uno de los países que conforman esta Comisión se reúnan periódicamente generando efectivamente un espacio de diálogo entre instituciones de ambos países, la sociedad civil (autoridades indígenas) y el sector privado. La continuidad en el proceso y el apoyo logístico que da específicamente la UICN han sido claves para esto. Por otra parte, este espacio ha permitido la coordinación de actividades e intercambios como fue el caso de los festivales ligados a la producción transfronteriza de cacao. Igualmente, la Comisión ha reforzado las relaciones de coordinación y cooperación de las instituciones que se encargan de la gestión del riesgo, como la Comisión de Emergencia de Costa Rica y el Sistema Nacional de Protección Civil de Panamá (sinaproc), los cuales cuentan con ferias informativas y un sistema de alerta y atención conjunta.

Declaraciones recientes de funcionarios del Ministerio de Planificación Nacional y Política Económica (MIDeplan) ${ }^{14}$ evidenciaron que la Comisión está viviendo un proceso de replanteamiento, debido a que en el Convenio suscrito por los gobiernos de Costa Rica y Panamá para el desarrollo fronterizo firmado en I995 no le asignaba recursos por ley a la Comisión Transfronteriza de la Cuenca del Sixaola. Es por esto que desde este ministerio se está intentando formalizarla, para repensar su misión y dotarla de un presupuesto con el fin de poder mantenerla en el tiempo.

\section{Representatividad y legitimidad}

Según la GIRH, todo usuario del agua puede ser considerado como un stakeholder o parte interesada, por lo que un principio puede participar en los procesos de negociación. Sin embargo, en la práctica muchos autores afirman que ese propósito es imposible (Warner y Simpungwe, 20I2: II2) y es por esto que su representatividad siempre será cuestionada. Conviene por lo tanto interrogarnos sobre quiénes tienen derecho a participar y por qué.

En el caso de la свGCs, los miembros son seleccionados por los secretarios de la Convención Binacional Costa Rica y Panamá (funcionarios públicos). La conformación de esta Comisión la define su reglamento; sin embargo, cabe recalcar

${ }^{14}$ Entrevista con Óscar Méndez, coordinador del área de Cooperación Internacional en noviembre 2018. 
que este no fue definido por los gobiernos sino por la UICN. La participación a esta instancia es restringida y los actores que intervienen deben ser reconocidos como representantes de instituciones o de organizaciones sociales (autoridades indígenas, sindicatos, etc.). Y a pesar de los esfuerzos por hacer de esta PMA un espacio representativo y participativo, pudieron identificarse numerosas críticas en el sentido de que siempre son "las mismas personas" las que participan tanto en la Comisión como en los proyectos establecidos por la UICN y el BID. Otros entrevistados pusieron en entredicho la legitimidad de algunos representantes de la sociedad civil, afirmando que no fueron elegidos de forma representativa. Actores considerados como "radicales" o "contestatarios" o "conflictivos" no son convocados a participar en estos espacios; es el caso del proyecto de kioscos socioambientales de la Universidad de Costa Rica y de ciertos grupos indígenas, lo que promueve una especie de diálogo sin adversarios. Estos actores son profundamente críticos puesto que para ellos estas PMA y los proyectos de cooperación no están abordando los verdaderos problemas de la cuenca, como es la contaminación por monocultivos, y se preguntan cómo se puede hablar de gestión integrada de la cuenca sin que se enfrente la problemática de los monocultivos.

Es así como a pesar de que estas comisiones buscaban motivar una participación más activa, en la realidad promueven una participación selectiva que no incorpora a la gran diversidad de actores de la cuenca ni incluye en su agenda las problemáticas identificadas por los actores como prioritarias.

Las decisiones generadas, al no ser el resultado de un proceso fundado en un debate participativo, no son consideradas como legítimas por muchos actores de la cuenca, por lo que son decisiones provisorias y revocables. Esto se debe a que las decisiones que resultan de estas plataformas son negociadas por minorías, a diferencia de las leyes que son votadas por un principio de mayoría (Hermet et al., 2005: 10). Representan de esta forma los intereses de algunos, de un grupo cerrado, jerárquico y reducido de actores que generalmente forman parte de las elites locales. Elites locales educadas que disponen de los conocimientos necesarios y de la experiencia para fungir como intermediarios de los proyectos (Rodríguez, 2014:444). Pudimos observar, al analizar las listas de participación facilitadas por los distintos proyectos, que generalmente son los mismos actores los que participan de forma repetitiva en los eventos convocados por los diversos proyectos. Se podría así afirmar que estas plataformas están al servicio de los proyectos, ya que a través de ellas las organizaciones como la UICN y el BID legitiman y validan sus propias intervenciones en los territorios en los que ejecutan los proyectos y frente a los donantes. 
Adicionalmente, la agenda tratada por estas PMA responde a las iniciativas y los intereses de los proyectos de estas organizaciones internacionales. La participación está reducida a un grupo limitado de actores que son generalmente cercanos ideológicamente a la UICN o al BID. Eso limita la posibilidad de desarrollar un verdadero debate, ya que los participantes generalmente se adhieren a las agendas y los intereses de estas organizaciones internacionales.

En este sentido, Hermet muestra que en este tipo de plataformas hay una ausencia de participación ampliada y afirma que "los mecanismos de gobernanza limitan la participación a actores o agentes directamente asociados en el proceso [y] excluyen a los grupos radicales para evitar los conflictos" (Hermet, 2005: II).

En cuanto a la efectividad de esta Comisión, la presente investigación permitió determinar que los resultados tangibles son bastante limitados, puesto que las decisiones que genera no pueden ser impuestas jurídicamente. Las decisiones son recomendaciones no obligatorias formuladas en función de las agencias de cooperación o desde los organismos internacionales, por lo que la aplicación de estas acciones queda sujeta a la buena voluntad de los involucrados (Warner y Simpungwe, 20I2: 127).

La falta de autoridad conjugada a la ausencia de recursos propios de estas PMA hacen que esta instancia sea particularmente ineficiente. Además, estudios de PMA en la gestión de recursos naturales demuestran que estas resuelven rara vez los conflictos y tienen un nivel de influencia bastante débil. A pesar de esto, cabe destacar que los actores entrevistados afirmaron que si bien el impacto de estas PMA es cuestionable, estas funcionan como espacios de formación política para los actores locales, lo cual ha generado un capital social que se ha involucrado posteriormente en otros procesos de incidencia.

La centralidad dada a la GIRH y a la cuenca como unidad de gestión por estos proyectos de cooperación les resta protagonismo a las verdaderas problemáticas socioambientales. Se impone así una agenda global que, si bien es también importante, no está tomando en cuenta las necesidades inmediatas de las poblaciones locales. Esto genera que los actores locales tengan una participación poco constante, una vez que los proyectos se retiran de los territorios, y generalmente estas PMA tienden a desaparecer. 


\section{Dispositivos deliberativos ‘ ¡más democracia o más bien un pluralismo limitado?}

El Banco Mundial presenta a la gobernanza como un forma "avanzada de democracia, de participación, de proximidad con los ciudadanos, de eficiencia y de autogobierno (self rule) (Kazancigil, 2005: 54); sin embargo, en la práctica la relación entre la gobernanza, el Estado y la democracia es mucho más compleja.

En teoría los dispositivos deliberativos de gobernanza como las pMa promueven la democracia deliberativa. Según J. Warner y E. Simpungwe, las pma invitan a representantes de diversos intereses no desde una base de proporcionalidad, sino más bien a partir de una base identitaria. Las plataformas deliberativas son vistas como una forma de profundizar la democracia (Warner y Simpungwe, 20I2: II2). No obstante, la democracia está fundamentada en la soberanía popular que le delega al Estado la autoridad para gestionar el territorio y los recursos siguiendo del interés general. El Estado tiene el derecho de actuar en nombre de todos. Ante esto, podemos cuestionarnos: ¿son las PMA dispositivos democráticos en su funcionamiento interno? Y de forma más general, ¿fortalecen la democracia?

Ya demostramos que desde el punto de vista de su representatividad las PMA han suscitado muchas críticas, especialmente porque sus miembros son escogidos por los expertos internacionales en función de su proximidad ideológica. Los actores que participan no representan a una mayoría de ciudadanos. Para G. Hermet esto se asemeja al "sufragio censitario", ya que los que participan no fueron elegidos democráticamente (Hermet, 2005: 1I). También conviene mencionar que los estudios sobre las pMa en el mundo han demostrado que en materia de gestión del agua hay una ausencia de actores considerados como "los más pobres" en la estructura de las PMA, por lo que hay un monopolio del poder por parte de las elites locales y administrativas (Warner y Simpungwe, 2012: 113).

En cuanto a su representación, conviene preguntarnos sobre la no participación de actores considerados adversarios, como lo subraya A. Kriegg-Planque. Existe una tendencia a invisibilizar las tendencias contrarias y ocultar así los verdaderos conflictos, lo que puede generar la despolitización y la desideologización de los procesos de negociación (Krieg-Planque, 20I0ः 93). En fin, los dispositivos deliberativos como las PMA, destinadas a promover "buenas prácticas" según los arquetipos de la gobernanza, transfieren agendas, conceptos y estrategias, pero también una ideología neoliberal (Alphandery et al., 20I2: 13). Esto se puede observar específicamente en el lugar que se le asigna al Estado en las PMA, ya que estas muchas veces ponen en duda la capacidad de gestión del Estado y lo presentan como un actor más entre los otros. 
Se podría afirmar que las PMA se presentan como una solución, es decir, como instancias eficaces y vectores de articulación de grupos claves, frente a un Estado que es presentado como "un freno debido a su ineficiencia" (Jaglin, 2007: 275), sobre todo en territorios fronterizos en donde históricamente su presencia ha sido débil.

El Estado se banaliza, pierde su rol hegemónico y es presentado como un actor entre otros, que debe negociar en igualdad de condiciones con otros actores de la sociedad civil y actores privados (Chevallier, 2003: 207).

Es importante recalcar que estas PMA no pueden sustituir al Estado. Las organizaciones internacionales que las convocan hablan en el nombre de grupos que nunca las eligieron para representarlos (Papadopoulos, 2012: 6). Aun cuando en lo interno de estas PMA, actores elegidos negocian con actores no elegidos, estas comisiones no pueden remplazar al Estado. El Estado representa la soberanía popular y tiene por lo tanto la autoridad para gestionar el territorio y los recursos siguiendo el principio del bien común. El Estado actúa en nombre de todos, pero no es el caso de las organizaciones internacionales ni de los actores privados, los cuales representan sectores (sector ecologista, sector bananero, sector productor de piña, entre otros). Esto nos permite concluir que las PMA, al representar intereses de sectores específicos, promueven la gestión de bienes comunes de forma sectorial y su falta de representación pone en práctica un pluralismo limitado.

Si la Comisión de la Cuenca del Sixaola como pma no es representativa y no está asumiendo las acciones necesarias para hacerle frente a la contaminación y a la expansión de los monocultivos, podríamos cuestionarnos sobre el papel del Estado costarricense. Si bien es el responsable de velar por el bien común y el ambiente en esta región fronteriza, debemos cuestionarnos sobre sus estrategias de intervención y de control.

A partir de las entrevistas realizadas se pudo constatar que si bien estas PMA funcionan gracias al financiamiento y a la asistencia técnica de organizaciones internacionales, lo hacen también porque los Estados y sus instituciones públicas lo permiten. Estamos así frente a procesos desregulados, en donde el Estado cede voluntariamente a estas Comisiones la gestión de la cuenca.

$\mathrm{Al}$ conversar con los actores locales, en especifico representantes de la asociación de mujeres productoras de cacao orgánico ACOMUita, ellas afirmaban que el"Estado está pero no está". Si bien el Estado no está generando acciones de control y desarrollo en la cuenca, tuvo un rol central en la creación de condiciones necesarias como infraestructura y seguridad jurídica, para promover los emprendimientos privados, específicamente los relacionados con la explotación de banano y plátano, delegando a 
estas estas organizaciones internacionales la responsabilidad de garantizar la gestión ambiental de la cuenca a través de la Comisión Binacional.

\section{Algunas conclusiones}

La Comisión para la Gestión de la Cuenca del río Sixaola es una pma que representa a "círculos cerrados" que son escogidos por actores exógenos para gestionar de forma sectorial un bien común. Podríamos así afirmar que esta PMA tiene una legitimidad política limitada, ya que no es representativa y los actores que participan fueron escogidos por su proximidad ideológica limitando el debate público a otros actores que tienen posiciones divergentes. Además, la soberanía popular no interviene, ya que estos no fueron elegidos (Hermet et al., 2005: 9). Un verdadero proceso democrático tiene, por el contrario, como fundamento la soberanía popular, la cual le delega al Estado la autoridad para gestionar su territorio y sus recursos siguiendo el interés general.

A pesar de las debilidades de esta PMA y de los proyectos, es importante reconocer el rol que juegan estos organismos y ong internacionales. Se debe reconocer que estas regiones fronterizas se han beneficiado de la inversión que estos proyectos han generado. Podemos concluir que los actores exógenos tienen una importante influencia (recursos, equipos técnicos y estrategias de intervención) en la gestión ambiental de las regiones fronterizas, y muchas veces han venido a llenar algunos "vacíos" históricos e institucionales dejados por el aparato gubernamental en estas áreas. También han sacado provecho de la particularidad de estas regiones fronterizas para poner en práctica sus proyectos debido a que en estas zonas llegan a contar con mayor autonomía con respecto a otros territorios en donde la presencia de los Estados es más importante. Sin embargo, existen criticas serias a la efectividad y legitimidad de muchas de sus estrategias de intervención.

La debilidad de la PMA estudiada es evidente, ya que excluye de los espacios de negociación a los actores contestatarios e ignora los conflictos socioambientales alrededor de los monocultivos. Además, los actores locales entrevistados las perciben muchas veces como impuestas desde "afuera". Otro punto clave es que introducen a actores privados en los procesos de negociación ligados a la gestión de bienes comunes.

No obstante, para Papadóupulos estas debilidades podrían ser superadas si las PMA garantizaran mayor representatividad, una participación equitativa, transparencia y rendición de cuentas y colaboraran con las instituciones democráticas. 
Con una nueva reconfiguración las PMA podrían reforzar los espacios de toma de decisión, fungiendo como instancias complementarias a las propuestas por la democracia participativa a escala local (gobiernos locales), siempre y cuando se respete la primacía de las instituciones públicas.

En cuanto a su agenda de trabajo, cabe destacar que tanto esta PMA como los proyectos que se desarrollan en esta frontera proponen acciones de reforestación, de capacitación en GIRH, de adaptación al cambio climático, pero no proponen ni mencionan el tema de la contaminación generada por la expansión de monocultivos de banano y plátano considerados por los actores locales entrevistados como la principal causa de contaminación en esta cuenca. Ninguna acción es propuesta para hacerle frente ni a su expansión ni a sus impactos (contaminación, sedimentación, deforestación, explotación laboral, etc.) por parte de esta PMA o de estos proyectos.

Después del estudio de los proyectos y las acciones de esta pMa podemos afirmar que el ignorar los conflictos socioambientales es una decisión política voluntaria. Para entender por qué las organizaciones internacionales presentes en esta frontera toman distancia con respecto a estas problemáticas ambientales, conviene estudiar la naturaleza de las organizaciones y el origen de los fondos, para así entender cómo funcionan sus lógicas de acción. Primero que todo, estas organizaciones tienen sus propias agendas, que deben de ser aplicadas a escala local. Estas agendas son pensadas desde un sistema internacional jerarquizado que podemos llamar "oligopolio de la conservación"(Dumoulin y Rodary, 2005), que rara vez toma en cuenta las particularidades de los sitios en donde ejecutan sus proyectos. Igualmente, estos proyectos responden a las agendas de las agencias internacionales de cooperación que financian sus acciones. W. Robinson afirma que la principal causa del "boom" de las ong es que los Estados del norte las financian (Robinson, 2003: 227). Los recursos de estas organizaciones vienen de gobiernos, instituciones financieras y empresas del norte, y esto genera una transferencia vertical de agendas y de metodologías de norte a sur. La proximidad de estas organizaciones internacionales con respecto a los gobiernos y las empresas que muchas veces las financian a través de fundaciones genera una "superposición institucional" que hace muchas veces muy difícil distinguir quién es quién ( Robinson, 2003: 227).

Para W. Robinson, la ayuda al desarrollo ha sido siempre un instrumento de los poderes hegemónicos para abrir nuevos mercados, facilitar el acceso a los recursos naturales e integrar regiones "poscoloniales" en la economía mundo capitalista (Robinson, 2003: 227). Para este autor, esto no es cuestión de caridad o filantropía sino más bien de un proceso de privatización: los fondos ya no son dados a los 
Estados, sino a los grupos "no gubernamentales" contratados para que ejecuten acciones que deberían ser realizadas por los Estados.

Las ONG internacionales y la PMA estudiadas mantienen su distancia con los movimientos y actores considerados "radicales"; no defienden a los sectores populares y representan más bien los intereses de las elites locales-trasnacionales. Como afirman Chartier y Ollitrault, estas organizaciones están determinadas por las alianzas que han suscrito y por sus fuentes de financiamiento. Para mantener una posición privilegiada requieren mantener su credibilidad y su perfil (neutral, interés general, respecto a principios democráticos), por lo que sería autodestructivo para ellas alejarse radicalmente de los registros de acción dominantes (Chartier y Ollitrault, 2005:36).

En efecto, las organizaciones internacionales no son autónomas y están determinadas por los intereses de los gobiernos, de las elites políticas locales-nacionales y de las empresas. Esto puede explicar su falta de toma de posición con respecto a los conflictos socioambientales ligados a la expansión de los monocultivos. La PMA estudiada trata temas menos controversiales, menos políticos, menos ideológicos, es decir, menos conflictivos. Tiene así una tendencia, como afirma Robinson, a "despolitizar la sociedad" y extraer la "ideología" de los debates. El tema de la expansión de los monocultivos nos permite observar este proceso de desideologización, ya que los monocultivos constituyen una problemática que supone una toma de posición con respecto a los modos de producción capitalistas que generan importantes dinámicas de despojo en estas comunidades. Ignorando este tema, las organizaciones no gubernamentales aceptan el modelo de desarrollo capitalista basado en la lógica de acumulación por desposesión que este tipo de agricultura motiva (Harvey, 2004). La acumulación por desposesión, según D. Harvey, se refiere a la transferencia de activos a través de una gran variedad de procesos económicos, de los propietarios domésticos hacía otros que son generalmente actores exógenos que se apropian de dichos activos y los acumulan (Harvey, 2004: II4). La mercantilización y privatización de la tierra, la supresión del derecho a los bienes comunes, el desplazamiento de poblaciones campesinas e indígenas y la apropiación de activos son solo ejemplos de este proceso (Harvey, 2004: I13).

El papel del Estado es determinante, ya que actúa como socio o facilitador de estos procesos, sin asumir realmente un rol redistributivo o de control. Tal como afirma Cáceres (2015: I17-I18), si el Estado no asume un papel activo en la regulación de la conservación de la naturaleza, el capitalismo no va a regularse a sí mismo. Al disponer de marcos normativos y sociales que permiten la expansión de estas 
actividades, el Estado también puede no solo ser cómplice de la acumulación por desposesión, sino también crear nuevas formas de despojo.

Podemos así concluir que estas organizaciones y PMA no buscan generar cambios estructurales ni poner en duda el orden establecido. Estas organizaciones no son movimientos sociales, sino alternativas a ellos, pensadas por un oligopolio de la conservación al servicio de un sistema capitalista (Robinson, 2003: 228).

\section{Bibliografía}

Aguilar, Grethel A. y Alejandro Iza

2009 Gobernanza de aguas compartidas: aspectos jurídicos e institucionales, uicn, Oficina Regional de Mesoamérica.

Alphandery, Pierre, Marcel Djama, Agnès Fortier y Ėve Fouilleux

2012 Normaliser au nom du développement durable, Editions Quae, pp. I93. Amilhat-Szary, A. L.

2009 "La prise de position des acteurs privés dans la gouvernance territoriale: enjeux, opportunités, risques", en Les acteurs privés dans la gouvernance: firme et territoires en Amérique Latine, Francia, Anne-Laure Amilhat-Szary, pp. 6-17

Amilhat-Szary, Anne Laure y Marie-Christine Fourny

2006 Après les frontières, avec la frontière: nouvelles dynamiques transfrontalières en Europe, Francia, Éd. de l'Aube. https://halshs.archives-ouvertes.fr/ halshs-00377502/document

Bossy, Thibault y Evard Aurélien

2010 "Communauté épistémique", en Dictionnaires des politiques publiques, Francia, Presses de Sciences Po., pp. 140-4I.

Bouquet, Christian

2012 "La GIRE: Un modèle difficile à transférer en Afrique subsaharienne", en La gestion intégrée des ressources en eau en Afrique subsaharienne: Paradigme occidental, pratiques africaines, Quebec, Presses Universitaires de Québec, pp. 55-73.

Cáceres, Daniel M.

2015 "Accumulation by Dispossession and Socio-Environmental Conflicts Caused by the Expansion of Agribusiness in Argentina", Journal of Agrarian Change, I5, pp. II6-I47. DoI: https://doi.org/IO.III/joac.I2057 
Chartier, Denis y Sylvie Ollitrault

2005 "Les ONG d'environnement dans un système international en mutation: des objets non identifiés?", en Représenter la nature? oNG et biodiversité, París, Catherice Aubertin, pp. 2I-58.

Chevallier, Jacques

2003 "La gouvernance, un nouveau paradigme étatique ?", Revue française d'administration publique, 105-106(I), pp. 203-217. DOI: https://doi.org/I0.3917/rfap.105.0203

Dore, Jason, Louis Lebel, y François Molle

2012 "A framework for analyzing transboundary water governance complexes, illustrated in the Mekong Region", Journal of Hydrology, 466-467, pp. 23-36.

Dumoulin, David y Estienne Rodary

2005 "Les ONG, au centre du secteur mondial de la conservation de la biodiversité, en Catherine Aubertin, Représenter la nature? oNG et biodiversité, Marsella, IRD Editions, disponible en <http://books.opendition.org/ irdeditions/5642>, pp. 59-99.

Duncan, Quince y Carlos Meléndez

I972 El Negro en Costa Rica, San José, Costa Rica, Editorial Costa Rica.

Fourny, Marie-Christine

2005 "De la frontière naturelle à la nature comme lien transfrontalier. Du rôle et de la place de l'environnement et du milieu dans les coopérations transfrontalières", Tropisme des frontières, Approche multidisciplinaire, vol. I, París, Velasco-Graciet H. \& C. Bouquet.

Franklin, Hendrik

2007 "Costa Rica-Panamá, Gestión Integrada de ecosistemas en el río Sixaola", documento de proyecto, Banco Interamericano de Desarrollo.

Ghiotti, Stéphane

2014 "Le bassin transfrontalier. Emergence, contruction et intinéraire d'un concept", Regions E Cohesion, 4(2), pp.I0-29. DoI: https://doi.org/IO.3167/reco.2014.040202

Girot, Pascal y Carlos Granados

1997 "La cooperación transfronteriza y los nuevos paradigmas de la integración centroamericana," en Las fronteras del istmo: Fronteras y sociedades entre el sur de México y América Central, México, Bovin P., pp. 289-310. 
Global Water Partnership (GWP), Parténariat mondial de l'eau 2000 La gestion intégrée des ressources en eau, Estocolmo, GWP, https://www. gwp.org/globalassets/global/toolbox/publications/background-papers/o4-integrated-water-resources-management-2000-french.pdf

Haas, Peter

2013 "Epistemic Communities", en The Oxford Companion to Comparative Politics, vol. I, Londres, Oxford University Press, pp. 351-359.

Harvey, David

2004 "El'nuevo' imperialismo: acumulacion por desposesion", Socialist register, pp. 99-I29.

Hermet, Guy

2005 "La gouvernance serait-elle le nom de l'après démocratie? L'inlassable quête du pluralisme limité, en Guy Hermet, Ali Kazancigil y Jean François Prud'homme, La gouvernance: Un concept et ses applications, París, Karthala, pp. 17-47

Jaglin, Sylvy.

2007 "I2. La participation au service du néolibéralisme? Les usagers dans les services d'eau en Afrique subsaharienne", La Découverte, pp. 27I-29I.

Julien, Frédéric.

2012 "Leau à la crise mondiale, solution mondiale?", en La gestion intégrée des ressources en eau en Afrique subsabarienne: Paradigme occidental, pratiques africaines, Quebec, Presses de l'Université du Québec, pp. I-I4.

Kauffer, Edith

2006 El agua en la frontera sur de México: una aproximación a la problemática de las cuencas compartidas con Guatemala y Belice, AHA-CIESAs-CONAGuA-Colsan-Center for U.S.-Mexican Studies, México, pp. 22-29.

Kazancigil, Ali

2005 "La gouvernance et la souveraineté de l'Etat", en Guy Hermet, Ali Kazancigil y Jean François Prud'homme, La gouvernance: Un concept et ses applications, París, Karthala pp. 49-64.

Krieg-Planque, Alice

2010 'La formule développement durable' : un opérateur de neutralisation de la conflictualité", Langage et société, 134(4), pp.5-29. DoI: https://doi.org/I0.3917/ls.134.0005 
Llaguno, José, Julián Llaguno, Sindy Mora Solano, Ana Lucía Gutiérrez Espeleta, Priscilla Barrios Alfaro y Flavio Mora Moraga

2014 "Políticas y conflictos socio ambientales: El caso de la Tenencia de la tierra y los monocultivos En el Caribe de Costa Rica (2006-2012)". Revista de Ciencias Sociales, I45, pp. 8I-98, ISSN 0482-5276.

McSweeney, Kendra, Nazih Richani, Zoe Pearson, Jennifer Devine y David J. Wrathall

2017 "Why Do Narcos Invest in Rural Land?", Journal of Latin American Geography, 16(2), pp.3-29. DOI: https://doi.org/I0.1353/lag.2017.0019

Medina, Lucile

2009 "Les frontières de l'isthme centraméricain, des marges symboliques à des espaces en construction", Espaces et Sociètés, I38, pp. 35-50. DOI: https://doi.org/I0.3917/esp.138.0035

Meyer, Morgan y Susan Molyneux-Hodgson

20II 'Communautés épistémiques': une notion utile pour théoriser les collectifs en sciences?", Terrain et Travaux, I8, pp. I4I-154.

Molle, François

2012 "La GIRE : anatomie d'un concept". en Gestion intégrée des ressources en eau : paradigme occidental, pratiques africaines, en Julien (ed.), Géographie Contemporaine, Quebec, Presses de l'Université du Québec, pp. 23-53.

Papadopoulos, Yannis

2012 "The democratic quality of collaborative governance", en The Oxford Handbook of Governance, Londres, D. Levi-Faur.

Robinson, William I.

2003 Transnational conflicts: Central America, social change and globalization, Londres, Verso.

Rodríguez, Tania

2014 "Gouverner l'environnement dans des régions frontalières. Coopération et conflits dans les bassins du fleuve San Juan (Costa Rica-Nicaragua) et du fleuve Sixaola (Costa Rica-Panama)", tesis inédita de doctorado en Geografía, París, Universidad de Paris vir-Paris Diderot, pp. 616.

Santos, Boaventura de Sousa

2007 "Más allá de la gobernanza neoliberal: el Foro Social Mundial como legalidad y política cosmopolitas subalternas", en El derecho y la globalización desde abajo: hacia una legalidad cosmopolita, Anthropos/Universidad Autónoma Metropolitana-Cuajimalpa, pp. 3I-60. 
Steins, Nathalie A. y Victoria M. Edwards

I999 "Platforms for Collective Action in Multiple-Use Common-Pool Resources", Agriculture and Human Values, I6(3), pp. 24I-255. DoI: https://doi.org/10.1023/A:1007587330755

Warner, Jeroen y Eliab Simpungwe

2012 "La participation des usagers dans la question de l'eau. Quelles perspectives pour les plateformes multi acteurs en Afrique Subsaharienne", en La gestion intégrée des ressources en eau en Afrique subsabarienne: Paradigme occidental, pratiques africaines, Quebec, Presses Universitaires de Québec, pp. 107-I33

Wong, Carolina y Nazareth Porras

2013 "Reglamento de la Comisión Binacional de la Cuenca del Sixaola: Un instrumento para la sostenibilidad social y ambiental", UICN, https:// www.iucn.org/sites/dev/files/import/downloads/documento_reglamento_vesion_final_pdf, pp. I-I5.

Tania Rodríguez Echavarría

Doctora en Geografía de los países emergentes de la Universidad de Paris vir-Paris Diderot, Francia. Es docente e investigadora titular de las escuelas de Ciencias Políticas y de Geografía de la Universidad de Costa Rica. Sus líneas de investigación son las fronteras, los conflictos socio-ambientales y los procesos de cooperación transfronteriza. También investiga sobre el impacto de los monocultivos en la región centroamericana y la incursión de nuevos monocultivos verdes (cacao y otros). Entre sus más recientes publicaciones están "Entender el extractivismo en regiones fronterizas. Monocultivos y despojo en las fronteras de Costa Rica”, en la revista Sociedad y Ambiente, julio-octubre de 20I8, de El Colegio de la Frontera Sur, en coautoría con Alexa Obando y Marylaura Acuña, y“Extractivismo, petróleo y resistencia. El caso de'Acción de Lucha Anti-petrolera (adela)' en el Caribe sur de Costa Rica (1999-2002)", Revista Anuario de Ciencias Políticas, Centro de Investigación y Estudios Políticos, Universidad de Costa Rica. Y pendiente de publicación está el capitulo "Mitos y realidades de la gobernanza del agua en la cuenca transfronteriza del río Sixaola (Costa Rica-Panamá)", a publicarse en el libro Cuencas transfronterizas: la apertura de la presa del nacionalismo metodológico, Ciesas, México. 
Citar como: Tania Rodríguez Echavarría (2019),"Gobernanza ambiental en cuencas transfronterizas: la cuenca del río Sixaola (Costa Rica-Panamá)", Iztapalapa. Revista de Ciencias Sociales y Humanidades, núm. 87, año 40, julio-diciembre de 2019, ISSNः 2007-9176; pp.7I-99. Disponible en <http:// revistaiztapalapa.izt.uam.mx/index.php/izt/issue/archive >. 